\title{
Gastric Variceal Hemorrhage in a Noncirrhotic Patient Treated with Balloon-Occluded Retrograde Transvenous Obliteration
}

\author{
Jaap W. Ottevanger · Carla S. P. van Rijswijk • \\ Bart van Hoek • Mark C. Burgmans
}

Received: 9 May 2014 / Accepted: 24 May 2014/Published online: 3 July 2014

(C) Springer Science+Business Media New York and the Cardiovascular and Interventional Radiological Society of Europe (CIRSE) 2014

\section{Introduction}

We read with great interest the recent article by Saad et al. [1]. We fully agree with the authors that balloon-occluded retrograde transvenous obliteration (BRTO) is a valuable treatment that can be used as an alternative to transjugular intrahepatic portosystemic shunt (TIPS). BRTO is also an excellent treatment option in patients for whom TIPS would not be beneficial. Gastric varices most commonly occur in patients with portal hypertension due to cirrhosis. Yet gastric varices may also develop in patients without underlying liver cirrhosis, and TIPS may not be beneficial or possible in these patients. BRTO is preferred over TIPS in such cases. We present a case of BRTO in a patient with active hemorrhage from gastric varices that were due to post pancreatitis splenic vein occlusion.

\section{Case Report}

Approval by the institutional review board and informed consent are not required for anonymized case reports in our institution. A 62-year-old man with chronic pancreatitis

\footnotetext{
J. W. Ottevanger $(\bowtie)$ · C. S. P. van Rijswijk · B. van Hoek

M. C. Burgmans

Department of Radiology, Leiden University Medical Center, Albinusdreef 2, 2333 ZA Leiden, The Netherlands

e-mail: jaapottevanger@hotmail.com

C. S. P. van Rijswijk

e-mail: C.S.Pvan_Rijswijk@lumc.nl

B. van Hoek

e-mail: B.van_Hoek@LUMC.nl

M. C. Burgmans

e-mail: M.C.Burgmans@lumc.nl
}

was admitted to the gastroenterology department of another institution with severe hematemesis and melena. Medical history included diabetes mellitus type 2 and hypertension. The patient was admitted to the intensive care unit and subsequently intubated. Hemoglobin had dropped to $4.1 \mathrm{mmol} / \mathrm{L}$ (previously known normal value was $8.1 \mathrm{mmol} / \mathrm{L}$ ), and three units of packed red blood cells and three units of fresh frozen plasma were administered.

During the first few days, repeated gastroscopy performed in the referring hospital showed extensive clot formation in the gastric fundus without visualization of a possible active bleeding site. One day before transfer to our institution, gastroscopy revealed active hemorrhage from gastric varices, but Histoacryl injection was not considered to be feasible as a result of poor visualization of the bleeding focus. To control the bleeding, a Sengstaken-Blakemore tube was placed after each gastroscopy. A two-phase abdominal computed tomography (CT) with intravenous contrast was performed and revealed a gastrorenal shunt and active bleeding of gastric varices, signs of chronic pancreatitis, and splenic vein occlusion (Figs. 1 and 2). With the Sengstaken-Blakemore tube in situ, the patient was transferred to our hospital for further treatment.

He was hemodynamically stable on arrival at our hospital. Baseline laboratory values were as follows: ammonia $71 \mathrm{~g} / \mathrm{L}$, alkaline phosphatase $98 \mathrm{U} / \mathrm{L}$, gamma glutamyl transferase 90 $\mathrm{U} / \mathrm{L}$, aspartate transaminase $22 \mathrm{U} / \mathrm{L}$, alanine transaminase 22 $\mathrm{U} / \mathrm{L}$, lactate dehydrogenase $154 \mathrm{U} / \mathrm{L}$, amylase $30 \mathrm{U} / \mathrm{L}$, hemoglobin $6.2 \mathrm{mmol} / \mathrm{L}$, thrombocytes $83 \times 10^{9} / \mathrm{L}$, international normalized ratio 1.2 , and activated partial thromboplastin time $32.8 \mathrm{~s}$. It was decided to perform transjugular measurements of the hepatic vein pressure gradient and proceed with BRTO in the absence of portal hypertension.

The BRTO procedure was performed under general anesthesia. An 8F Berenstein occlusion balloon (Boston 

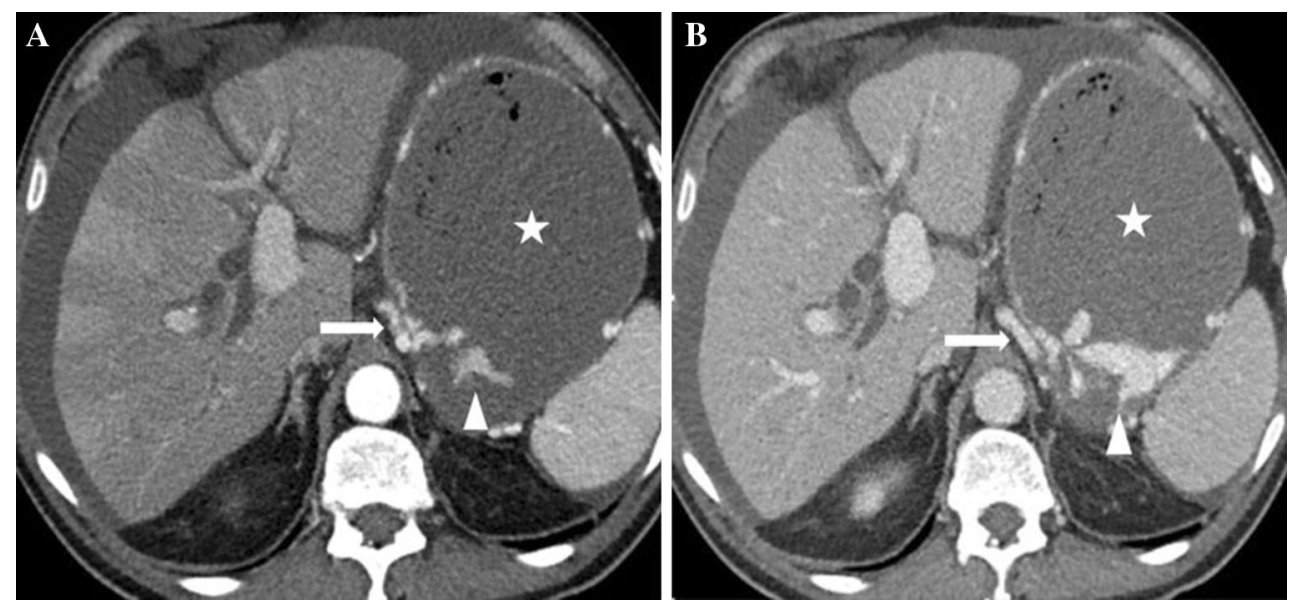

Fig. 1 Axial CT images in arterial phase (A) and portal venous phase (B) show gastric varices (arrows) with active bleeding (arrowheads) and blood-filled stomach (stars). Note the more prominent active bleed on the portal venous image (B)
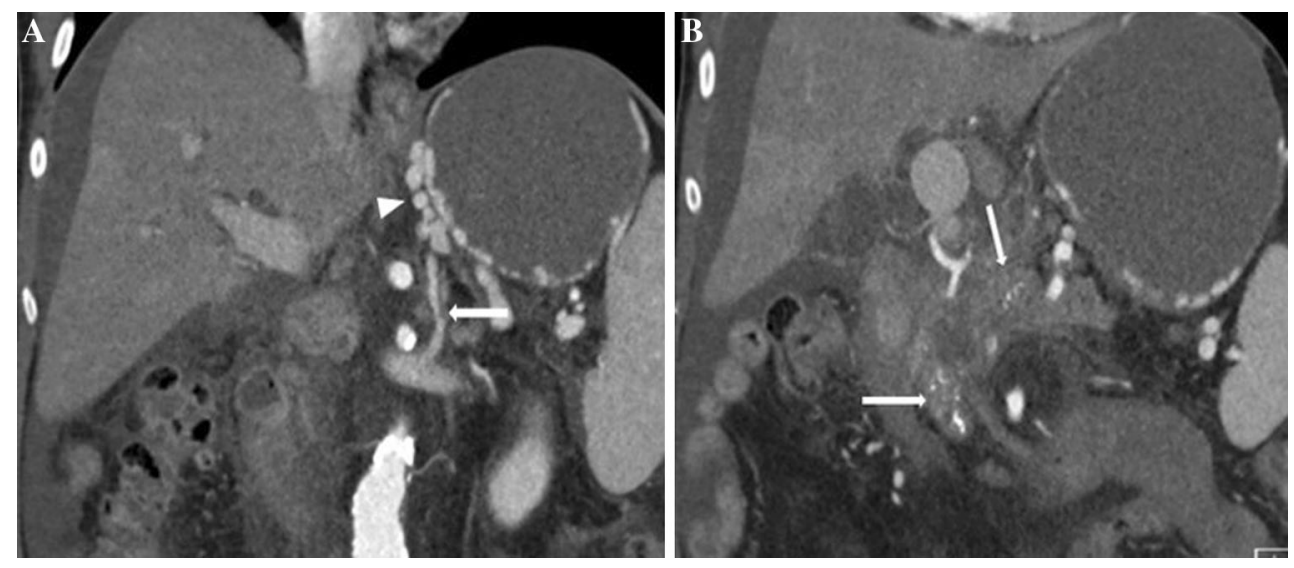

Fig. 2 Coronal CT images in portal venous phase show the gastrorenal shunt (arrow, A) and gastric varices (arrowhead, A). Pancreatic calcifications in the setting of chronic pancreatitis are seen in the pancreatic body and head (arrows, B)

Scientific, Natick, MA, USA) was introduced through a $8 \mathrm{~F}$ Brite-Tip vascular sheath (Cordis, Miami Lakes, FL, USA) placed in the right jugular vein and used to measure the hepatic vein pressure gradient. The hepatic vein pressure gradient was $3 \mathrm{~mm} \mathrm{Hg}$, and right-sided portal hypertension was thus ruled out.

Under ultrasonographic guidance, the right common femoral vein was punctured and a 9F Brite-Tip vascular sheath (Cordis) was introduced. The left renal vein and gastrorenal shunt were selectively catheterized using a $4 \mathrm{~F}$ multipurpose catheter (Cordis) and $0.035^{\prime \prime}$ stiff Terumo guide wire (Terumo, Tokyo, Japan). An 8F Berenstein occlusion balloon (Boston Scientific) was then inserted over the guide wire and into the gastrorenal shunt. Balloonoccluded venography revealed a large tortuous varix at the location of the lesser curvature of the stomach (Fig. 3). The left phrenic vein was catheterized using a $2.7 \mathrm{~F}$ Progreat microcatheter (Terumo, Tokyo, Japan) and selectively coiled with two diamond-shaped $3 \mathrm{~mm}$ microcoils (Boston
Scientific). A mixture of gelfoam, $6 \mathrm{~mL}$ of Ultravist 300 , $2 \mathrm{~mL}$ of lipiodol, and $6 \mathrm{~mL}$ of aethoxysclerol $1 \%(10 \mathrm{mg} /$ $\mathrm{mL}$ ) was injected through the occlusion balloon (Fig. 3). The occlusion balloon remained inflated in the gastrorenal shunt for $12 \mathrm{~h}$, after which it was removed under fluoroscopic guidance. No complications occurred during the procedure. A three-phase CT performed 2 days after the procedure showed successful occlusion of the embolized gastric varices and no signs of bleeding (Fig. 3). The patient was discharged from the hospital 1 week later without any further rebleeding and a hemoglobin of $7 \mathrm{mmol} / \mathrm{L}$ before discharge.

\section{Discussion}

Gastric varices most commonly occur in patients with portal hypertension due to cirrhosis. The patient we describe in this case report, however, had gastric varices 


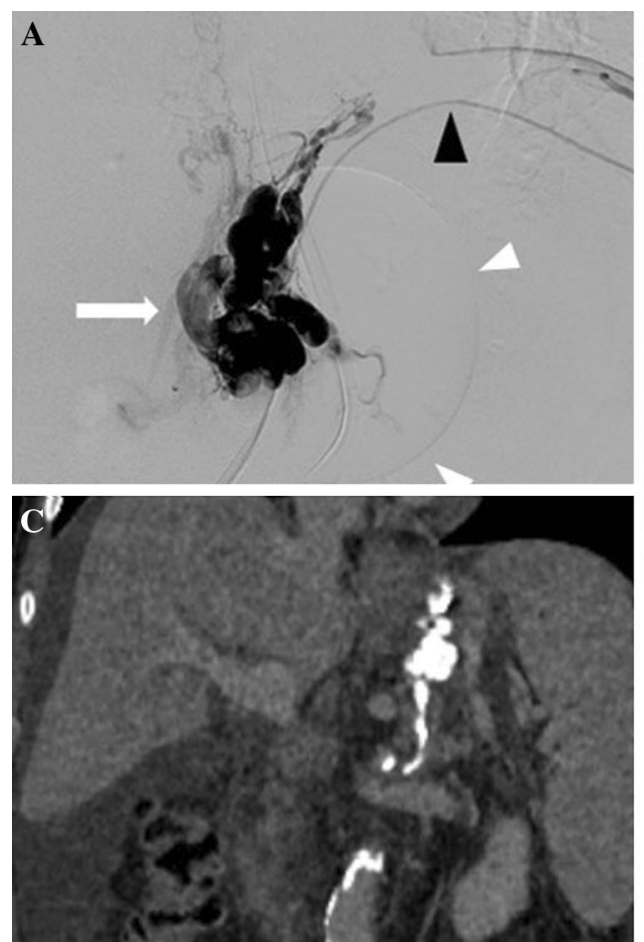

Fig. 3 Angiography before (arrow, A) and after (arrows, B) embolization of gastric varices and gastrorenal shunt. Left phrenic vein (black arrowhead, A) was embolized with coils (arrowhead, B). Sengstaken-Blakemore tube was placed to control bleeding (white

due to postpancreatitis splenic vein thrombosis, causing localized portal hypertension. In a study by Sarin et al. [2] including 568 patients with portal hypertension, liver cirrhosis was the cause of portal hypertension in $53.0 \%$ of patients. Other causes of portal hypertension were noncirrhotic portal fibrosis (20.2\% of patients), extrahepatic portal vein obstruction $(20.6 \%)$, and hepatic venous outflow obstruction $(3.5 \%)$. Of all patients in the study, $20 \%$ had gastric varices. The incidence of gastric varices in the patients with extrahepatic portal vein obstruction was higher $(38.5 \%)$ compared to patients with noncirrhotic portal fibrosis $(30.4 \%)$, cirrhosis $(21.6 \%)$, and hepatic venous outflow obstruction (5.7\%). A systematic review and meta-analysis by Butler et al. [3] showed that splenic vein thrombosis is frequently observed in the setting of pancreatitis (acute and chronic) and may lead to esophagogastric varices in many cases. Gastric varices are observed in $77.3 \%$ of patients with pancreatitis-induced splenic vein thrombosis, and the associated GI bleeding rate in patients with this thrombosis is as high as $12.3 \%$ [3]. Bleeding from gastric varices in patients with portal hypertension is less common than from esophageal varices but is associated with higher mortality rates [2]. Moreover, gastric varices seem to bleed at lower portal pressures than esophageal varices [4]. As mentioned by Saad et al. [1],

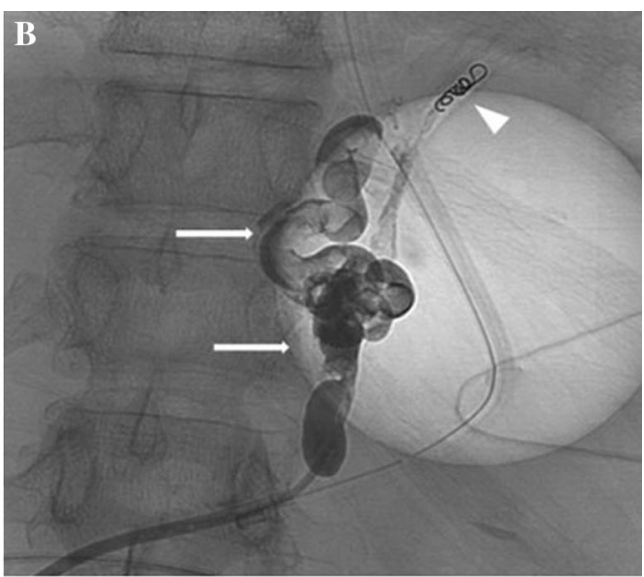

arrowheads, A). Corresponding coronal CT image 2 days after embolization indicates successful embolization of gastric varices and gastrorenal shunt $(\mathbf{C})$

endoscopic treatment of bleeding gastric varices is less effective compared to esophageal varices.

The first-line treatment for bleeding gastric varices is endoscopic injection sclerotherapy. When an endoscopic approach fails, the choices consist of TIPS or BRTO. In Western countries, TIPS is the preferred option because this deals with the underlying problem of portal hypertension [1]. However, in patients with gastric varices without right-sided portal hypertension, TIPS may not be effective in achieving decompression of the varices [5]. In such patients with left-sided portal hypertension, BRTO is the preferred therapeutic option, as it was in the case described. As highlighted by Saad et al. [1], a gastrorenal shunt may be absent in 15-16\% of patients with gastric varices. BRTO may only be possible in such cases using other collateral portosystemic pathways. Yet performing BRTO via such unconventional pathways is difficult and requires extensive BRTO experience. Alternatively, if endoscopic Histoacryl injection is not feasible, percutaneous transhepatic obliteration may be used to treat gastric varices in the absence of a gastrorenal shunt. This approach is technically less challenging and can also be attempted after unsuccessful BRTO [1, 6].

Both BRTO and TIPS are effective in treating gastric varices caused by portal hypertension. When gastric 
varices develop in the absence of right-sided portal hypertension-for example, as result of splenic vein thrombosis-TIPS may not be effective. Therefore, interventional radiologists should be familiar with and capable of performing BRTO.

Conflict of interest Jaap W. Ottevanger, Carla S. P. van Rijswijk, Bart van Hoek, and Mark C. Burgmans declare that they have no conflict of interest.

\section{References}

1. Saad WE, Simon PO Jr, Rose SC (2014) Balloon-occluded retrograde transvenous obliteration of gastric varices. Cardiovasc Intervent Radiol 37:299-315

2. Sarin SK, Lahoti D, Saxena SP et al (1992) Prevalence, classification and natural history of gastric varices: a long-term follow-up study in 568 portal hypertension patients. Hepatology 16:1343-1349

3. Butler JR, Eckert GJ, Zyromski NJ et al (2011) Natural history of pancreatitis-induced splenic vein thrombosis: a systematic review and meta-analysis of its incidence and rate of gastrointestinal bleeding. HPB 13:839-845

4. Tripathi D, Therapondos G, Jackson E et al (2002) The role of the transjugular intrahepatic portosystemic stent shunt (TIPSS) in the management of bleeding gastric varices: clinical and haemodynamic correlations. Gut 51:270-274

5. Saad WE, Darcy MD (2011) Transjugular intrahepatic portosystemic shunt (TIPS) versus balloon-occluded retrograde transvenous obliteration (BRTO) for the management of gastric varices. Semin Intervent Radiol 28:339-349

6. Arai H, Abe T, Takagi H, Mori M (2006) Efficacy of balloonoccluded retrograde transvenous obliteration, percutaneous transhepatic obliteration and combined techniques for the management of gastric fundal varices. World J Gastroenterol 12:3866-3873 